\title{
Relativistic Two-Body Processes in Axial-Charge Transitions
}

\author{
E.D. Izquierdo \\ Departamento de Física, Facultad de Ciencias Exactas y Naturales, \\ Universidad de Buenos Aires. Pabellón 1, Ciudad Universitaria, (1428) Buenos Aires, Argentina \\ G. Barenboim and A.O. Gattone \\ Departamento de Física, Comisión Nacional de Energía Atómica \\ Av. del Libertador 8250, 1429 Buenos Aires, Argentina
}

(December 19, 1994)

\begin{abstract}
We study the contribution of two-body meson-exchange processes to axial charge transitions for nuclei in the lead, tin and oxygen regions. We conduct calculations in the Dirac-Hartree (the Walecka model) and the relativistic Hartree (where the full one-nucleon-loop effects are included) approximations. We present results indicating that one- and two-body processes enhance the matrix elements of the axial-charge operator by some $(100 \pm 20) \%$ in all three regions studied. This agrees well with the fit of eighteen first-forbidden $\beta$ decay transitions conducted by Warburton in the lead region. We also discuss some sensitivities present in the calculation.
\end{abstract}

\section{INTRODUCTION}

The unexpected enhancement found in processes driven by the axial charge, namely the cases of first-forbidden beta decays in heavy nuclei [1] and the production of pions near 
threshold in $p p \rightarrow p p \pi^{0}$ reactions [2], has given a new impetus to the study of meson-exchange currents (MEC) in axial charge transitions. The findings are really remarkable. In the first process for example — already known to be strongly renormalized by the presence of MECthe results found by Warburton [1] exceeded the anticipated theoretical estimate by a factor of two. In the second - a new result in a region not available to experiment before - the data overestimate the theoretical predictions (only the one-body contribution) by approximately a factor of five. Despite this sizable difference between theory and experiment the first result is acknowledged as the most dramatic since the process of first-forbidden $\beta$-decay was thought to be (at least to a large extent) under theoretical control [3].

The subject of axial charge transitions becomes more relevant from the viewpoint of relativistic theories of the nucleus. Most of the explanations advanced so far to account for these enhancements (refs. [4.5] in the first case and [6] in the second) rely on the presence of scalar heavy-meson exchange between two nucleons. Diagrams containing a nucleonantinucleon pair originating (or decaying) into a scalar meson of $m_{s} \approx 500 \mathrm{MeV}$ using couplings from either the Bonn or Paris potentials seem to take care of the discrepancy between the theoretical prediction and the experimental result. These large Lorentz scalar potentials have long been a distinctive feature of relativistic calculations whose explicit need, however, had not shown up in the non-relativistic phenomenology of nuclear processes before. The calculations alluded to above (non relativistic) incorporate the scalar degree of freedom formally via meson-exchange currents.

The connection between non-relativistic pair-exchange currents and relativistic meanfield theories for isovector currents was studied by Delorme and Towner [7] in their paper of 1987 (in the case of isoscalar currents there were previous calculations by Arima et al. [8] and Blunden [9]). Pair graphs are shown in Fig.1 (diagrams (a) and (b)). The relativistic Hartree contribution is obtained by closing the line on nucleon 2 and is shown in diagrams (c) and (d) (diagram (d) is usually recognized as the "backflow" correction). The difference between both approaches is that whereas in the relativistic case the propagation of an antinucleon is included in the one-body Green's function, in the non-relativistic case it can 
only be incorporated as an exchange current. Delorme and Towner found in their calculations large enhancements due to the scalar-exchange terms. Their conclusion at the time - for axial-charge transitions - was that such enhancement (and along with it the relativistic mean-field theory) was hardly consistent with experiment. Quite oppositely, they noticed that the conventional theory of meson exchange with short range cut-offs gave reasonable agreements with the data (ignoring, of course, the contribution of pair diagrams). The more recent work of Warburton on the one hand and the results of Meyer et al. on the other, prompted a reanalysis of the existing calculations under the light of the new data. At the present time it is generally held that scalar heavy-meson exchanges can account for the discrepancies between the predictions and the actual data [10].

This situation opened up the ground for further exploration of the predictions of relativistic models of nuclear structure to axial-charge processes. Since the physical content of the two descriptions (non-relativistic vs. relativistic) is different from the outset, the individual contribution of one- and two-body diagrams is not anticipated to be similar. We already mentioned that the one-body relativistic calculation includes the non-relativistic two-body pair graphs. Conversely, the two-body contribution in the relativistic calculation sums diagrams (like the one shown in Fig.2) that are not included in the traditional mesonexchange calculations. Though, a priori, contributions of this type are small a comparision between both approaches will only be meaningful once full calculations of one and two-body processes are conducted within each framework.

With this in mind we study in this paper the contribution of two-body processes within the relativistic approach. We concentrate exclusively on first-forbidden beta decays in nuclei (oxygen, tin and lead) where the relativistic mean-field can be used reliably. Regardless of the model the symmetries must be upheld. In particular chiral symmetry, namely the use of soft-pion theorems and current algebra, imposes a strong renormalization of the matrix elements due to pion-exchange processes (particularly through the $\pi \rho$-exchange diagram.) This mechanism needs to be incorporated explicitly into relativistic calculations since, at the mean-field level, the pion is absent due to parity arguments. It is clear that a relativistic 
description of axial-charge transitions in nuclei requires to go beyond the mean-field. A first approximation to the problem calls for a mean-field calculation (impulse approximation) to describe the one-body processes and additionally a MEC calculation taking care of the "backflow" and pion-exchange processes. We notice that the results of this programme are not expected to be free from the usual ambiguities of pion couplings in relativistic theories (for a review of the current status see ref. [11]).

Recently, within the framework of a relativistic model (quantum hadrodynamics II) we analyzed axial transitions [12 in heavy nuclei using the scheme outlined above. We obtained first the one-body contribution given by graphs (c) in Fig. 1; next, the RPAtype vertex corrections (graphs (d) in the same figure) [13] and, finally we investigated the contribution of the pion-exchange currents [14] (with no exchange diagrams.) Inspection of the results arrived at in this study showed that: First, despite some ambiguities related to the strength of the $\rho \mathrm{N}$ coupling constant, the sum of one- and two-body diagrams added up to an enhancement which was comparable to that obtained in non-relativistic calculations. Second, the theoretical calculations could only be brought into agreement with the data when pseudovector $\pi \mathrm{N}$ coupling was used (otherwise the results were anomalously large). Third, two-body pion-exchange processes were indispensable to understand the axial-charge data. These conclusions were based on calculations conducted on two particular transitions, one in $\mathrm{Tl}$ and the other in $\mathrm{Pb}$, and were intended to give an idea of what should be expected for other transitions in this mass region. In this paper we extend and deepen the analysis of the previous works. On the one hand, we give a complete description of the two-body pion-exchange contribution to the axial charge from the point of view of relativistic hadron theories. On the other, we extend the results of [14] and present results of MEC contribution to axial charge matrix elements for tin and oxygen. The idea is to move to lighter nuclei to study mass dependence, and the influence of the scalar field in this contribution.

Besides this introduction the paper contains two other sections. Section $\mathbb{1}$, divided into two subsections, deals with the calculation of the matrix elements of the axial charge. In the first subsection we give some details about the hadronic model we employ and the way 
the axial two-body current is derived. In the second we describe at some length the scheme to carry out the actual calculation of the matrix elements of the current between the nuclear states. The last section contains a discussion of the results and of the potential ambiguities involved in the calculation. Finally we present some conclusions.

\section{MESON-EXCHANGE-CURRENT MATRIX ELEMENTS OF THE AXIAL CHARGE}

\section{A. Hadron model and currents}

At tree level the two-body isovector axial vector current is given by the sum of the four diagrams shown in Fig. 3. In the figure we have used the symbol $\varepsilon$ to denote the field operator mediating the weak current. Diagrams $a$ ) and b) turn out to be the most important since they involve pion exchanges which generate the longest-range exchange currents. The contribution of the other two diagrams (involving the $\Delta$ ) is known to be small [15].

The two-body current from diagrams $a$ ) and $b$ ) can be calculated using standard Feynman rules. As stated meson and baryon propagators derived from some baryonic Lagrangian are needed. Throughout the paper we use the quantum hadrodynamical model II (QHD-II) of Serot and Walecka [16]. It includes the nucleon fields, two isoscalar meson fields -the scalar $\sigma$ and the vector $\omega$ - and two charged isovector meson fields -the pseudoscalar $\pi$ and the vector $\rho$. The full Lagrangian expression is,

$$
\begin{aligned}
\mathcal{L}= & \bar{\psi}\left[i \gamma_{\mu} D^{\mu}-\left(m_{N}-g_{\sigma} \sigma\right)-g_{\omega} \gamma_{\mu} \omega^{\mu}-i g_{\pi} \gamma_{5} \vec{\tau} \cdot \vec{\pi}\right] \psi \\
& +g_{\rho}\left(\partial^{\mu} \vec{\pi} \times \vec{\pi}\right) \cdot \vec{\rho}_{\mu}+\frac{1}{2} g_{\rho}^{2}\left(\vec{\pi} \times \vec{\rho}_{\mu}\right) \cdot\left(\vec{\pi} \times \vec{\rho}_{\mu}\right)+\frac{1}{2}\left(\partial_{\mu} \sigma \partial^{\mu} \sigma-m_{\sigma}^{2} \sigma^{2}\right) \\
& -\frac{1}{4} F_{\mu \nu} F^{\mu \nu}+\frac{1}{2} m_{\omega}^{2} \omega_{\mu} \omega^{\mu}-\frac{1}{4} \vec{B}_{\mu \nu} \vec{B}^{\mu \nu}+\frac{1}{2} m_{\rho}^{2} \vec{\rho}_{\mu} \cdot \vec{\rho}^{\mu} \\
& +\frac{1}{2}\left(\partial_{\mu} \vec{\pi} \cdot \partial^{\mu} \vec{\pi}-m_{\pi}^{2} \vec{\pi} \cdot \vec{\pi}\right)+\frac{1}{2} g_{\sigma \pi} m_{\sigma} \sigma \vec{\pi} \cdot \vec{\pi}
\end{aligned}
$$

In Eq.(2.1) the neutral and charged vector meson field strengths are defined by,

$$
F^{\mu \nu} \equiv \partial^{\mu} \omega^{\nu}-\partial^{\nu} \omega^{\mu}
$$


and

$$
\vec{B}_{\mu \nu} \equiv \partial_{\mu} \vec{\rho}_{\nu}-\partial_{\nu} \vec{\rho}_{\mu}-g_{\rho}\left(\vec{\rho}_{\mu} \times \vec{\rho}_{\nu}\right)
$$

respectively. We have also made use of the covariant derivative defined as

$$
D_{\mu} \equiv \partial_{\mu}+i \frac{1}{2} g_{\rho} \vec{\tau} \cdot \vec{\rho}_{\mu}
$$

There is a Higgs-meson sector associated with the $\rho$-meson field which has been suppressed by taking the scalar Higgs mass to be very large.

The $\pi N$ coupling in the QHD-II Lagrangian is pseudoscalar. To account for the small pion scattering length in $\pi N$ interactions a $\sigma \pi \pi$ coupling term is added. As we will see below pseudoscalar coupling with the standard parameters gives very large predictions for the contribution of one- and two-body processes. As an alternative to the actual calculation we have considered substituting pseudovector for both pseudoscalar $\pi N$ and $\sigma \pi \pi$ couplings. We emphasize that despite the different predictions both couplings show the same correct behaviour in the low-energy limit (in the two-body case) and both satisfy the constraint of partial conservation of the axial current (PCAC).

The strong vertices $\pi N N, \rho N N$ and $\rho \pi \pi$ as derived from the Lagrangian of QHD-II are shown in Fig. 4. The weak vertices $\pi \varepsilon$ y $\pi \rho \varepsilon$ (see Fig. 5) cannot obtain directly from the QHD-II Lagrangian since it does not contain the weak interaction. The structure of the $\pi \varepsilon$ vertex is obtained from symmetry arguments in pion decay where,

$$
\Gamma_{\pi \epsilon}^{\mu a b}=\left\langle v a c\left|J^{A, \mu a}\right| \pi^{b}(q)\right\rangle=i f_{\pi} q^{\mu} \delta^{a b}
$$

The value of the constant, $f_{\pi}=0.93 \mathrm{GeV}$, is taken from experiment [18. For the $\pi \rho \epsilon$ vertex a similar argument leads to a vertex of the form,

$$
\Gamma_{\pi \rho \epsilon}^{\mu \nu a b c}=\left\langle\pi^{a}\left(k_{1}\right) \rho^{b}\left(k_{2}\right)\left|J^{A, \mu c}\right| v a c u u m\right\rangle=\epsilon_{a b c} T^{\mu \nu}
$$

where now the structure and content of the $T^{\mu \nu}$ tensor cannot be determined from Lorentz covariance only. However we will see below that the requirement of $P C A C$ on the two-body axial current suffices to define it. 
The propagators of the $\pi$ and $\rho$ fields appearing in QHD-II are of the Klein-Gordon type. With them and the vertices just defined we can obtain expressions for the contributions to the two-body axial vector current density following Feynman prescriptions for diagrams $a$ ) and $b$ ) in Fig. 3. For the case of pseudovector $\pi N$ coupling they read,

$$
\begin{aligned}
J_{\rho \pi}^{A, \mu c}= & -i \frac{g_{\pi} g_{\rho}}{4 M}\left[\tau_{1} \times \tau_{2}\right]^{c} \bar{\psi}\left(p_{1}^{\prime}\right) \gamma^{5} \gamma_{\lambda} k_{1}^{\lambda} \psi\left(p_{1}\right) \\
& \times \frac{1}{k_{1}^{2}-m_{\pi}^{2}} T^{\mu \nu} \frac{1}{k_{2}^{2}-m_{\rho}^{2}} \bar{\psi}\left(p_{2}^{\prime}\right) \gamma_{\nu} \psi\left(p_{2}\right)
\end{aligned}
$$

for diagram a) and,

$$
\begin{aligned}
J_{\rho \pi \pi}^{A, \mu c}= & -i \frac{f_{\pi} g_{\pi} g_{\rho}^{2}}{4 M}\left[\tau_{1} \times \tau_{2}\right]^{c} \bar{\psi}\left(p_{1}^{\prime}\right) \gamma^{5} \gamma_{\lambda} k_{1}^{\lambda} \psi\left(p_{1}\right) \frac{1}{k_{1}^{2}-m_{\pi}^{2}} \\
& \times q^{\mu}\left(q+k_{1}\right)^{\nu} \frac{1}{q^{2}-m_{\pi}^{2}} \frac{1}{k_{2}^{2}-m_{\rho}^{2}} \bar{\psi}\left(p_{2}^{\prime}\right) \gamma_{\nu} \psi\left(p_{2}\right) .
\end{aligned}
$$

for diagram $b)$.

The sum of (2.7) and (2.8) gives the full two-body (2B) isovector axial-vector current,

$$
\begin{aligned}
J_{2 B}^{A, \mu c}= & -i \frac{g_{\pi} g_{\rho}}{4 M}\left[\tau_{1} \times \tau_{2}\right]^{c} \bar{\psi}\left(p_{1}^{\prime}\right) \gamma^{5} \gamma_{\lambda} k_{1}^{\lambda} \psi\left(p_{1}\right) \frac{1}{k_{1}^{2}-m_{\pi}^{2}} \frac{1}{k_{2}^{2}-m_{\rho}^{2}} \\
& \times\left\{T^{\mu \nu}+g_{\rho} f_{\pi} \frac{q^{\mu}}{q^{2}-m_{\pi}^{2}}\left(q+k_{1}\right)^{\nu}\right\} \bar{\psi}\left(p_{2}^{\prime}\right) \gamma_{\nu} \psi\left(p_{2}\right)
\end{aligned}
$$

The quantity between brackets behaves as the effective $\pi \rho \varepsilon$ vertex with the tensor $T^{\mu \nu}$ still an unknown. This last difficulty can be overcome after enforcing the requirement of PCAC on two nucleons. This is equivalent to demand that the four-divergence of the full two-body current be equal to,

$$
q_{\mu} J^{A, \mu c}=i f_{\pi} \frac{m_{\pi}^{2}}{q^{2}-m_{\pi}^{2}} M^{c}(2 B)
$$

In (2.10), $M^{c}(2 B)$ is the $\pi$-absorption amplitude on two nucleons which obtains from the $\rho \pi \pi$ diagram (diagram $b$ ) in Fig. 3) by removing the external $\varepsilon^{5 \mu a}$ line. Thus from the figure,

$$
\begin{aligned}
M^{c}(2 B)= & -\frac{g_{\pi} g_{\rho}^{2}}{4 M}\left[\tau_{1} \times \tau_{2}\right]^{c} \bar{\psi}\left(p_{1}^{\prime}\right) \gamma^{5} \gamma_{\lambda} k_{1}^{\lambda} \psi\left(p_{1}\right) \\
& \times\left(q+k_{1}\right)^{\nu} \frac{1}{k_{1}^{2}-m_{\pi}^{2}} \frac{1}{k_{2}^{2}-m_{\rho}^{2}} \bar{\psi}\left(p_{2}^{\prime}\right) \gamma_{\nu} \psi\left(p_{2}\right)
\end{aligned}
$$


Substitution of (2.11) into the right hand side of (2.10) gives for the $T^{\mu \nu}$ tensor the expression,

$$
T^{\mu \nu}=-f_{\pi} g_{\rho}\left[g^{\mu \nu}+\frac{q^{\mu} k_{1}^{\nu}}{q^{2}-m_{\pi}^{2}}\right]
$$

thus fixing the strength at the $\rho \pi \varepsilon$ vertex. It is important to notice that in the soft-pion limit $\left(k_{1} \rightarrow 0\right)$, one obtains $T^{\mu \nu}=-f_{\pi} g_{\rho} g^{\mu \nu}$. This is the same strength obtained by taking the soft-pion limit in hard-pion models (see for example, Ivanov and Truhlik [19].)

Substituting $T^{\mu \nu}$ into the expression for the axial current and using the relation $f_{\pi} g_{\pi}=$ $g_{A} M$ (Goldberger-Treiman),

$$
\begin{aligned}
J_{2 B}^{A, \mu c}= & -i \frac{g_{A} g_{\rho}^{2}}{4}\left[\tau_{1} \times \tau_{2}\right]^{c} \bar{\psi}\left(p_{1}^{\prime}\right) \gamma^{5} \gamma_{\lambda} k_{1}^{\lambda} \psi\left(p_{1}\right) \frac{1}{k_{1}^{2}-m_{\pi}^{2}} \frac{1}{k_{2}^{2}-m_{\rho}^{2}} \\
& \times\left\{-g^{\mu \nu}+\frac{q^{\mu} q^{\nu}}{q^{2}-m_{\pi}^{2}}\right\} \bar{\psi}\left(p_{2}^{\prime}\right) \gamma_{\nu} \psi\left(p_{2}\right) .
\end{aligned}
$$

which is our final expression for the full two-body isovector axial-vector current. Inspection of the quantity between curly brackets shows that $J_{2 B}^{A, \mu c}$ satisfies $P C A C$ since,

$$
q_{\mu} J_{2 B}^{A, \mu c} \propto q_{\mu}\left\{-g^{\mu \nu}+\frac{q^{\mu} q^{\nu}}{q^{2}-m_{\pi}^{2}}\right\}=q^{\nu} \frac{m_{\pi}^{2}}{q^{2}-m_{\pi}^{2}} \underset{m_{\pi \rightarrow 0}}{\longrightarrow} 0
$$

Thus, it is clear that the two-body axial current derived from the Lagrangian (2.1) satisfies PCAC and has the correct behaviour in the soft-pion limit.

\section{B. Multipole decomposition}

Having studied the structure of the relativistic two-body current we concentrate next on the nuclear structure. Individual transitions in nuclei are more conveniently studied by performing a multipole decomposition of the current matrix elements. For axial-charge mediated processes selection rules [12] limit the contributing operators to the axial-Coulomb (or charge) operator, and the polar-vector longitudinal and transverse-electric operators. The last two affect only minimally the total matrix element and therefore, though they will be taken into account in the actual calculations and displayed in the results, for the purpose

of this section we shall work exclusively with the first one. The axial-Coulomb operator is written in the form, 


$$
\hat{\mathrm{C}}_{J M}^{2 B}(q) \equiv \int d^{3} x M_{J}^{M}(q \vec{x}) J_{0}^{2 B}(\vec{x})
$$

with

$$
M_{J}^{M}(q \vec{x})=j_{J}(q x) Y_{J}^{M}(\hat{x}) .
$$

The reduced two-body matrix element of any multipole operator may be decomposed as a sum of products of a matrix element of the operator between two-particle states times a two-body density matrix element which accounts for the complexity of the many-body problem. Thus, for the axial Coulomb operator we shall write,

$$
\left\langle J_{f}\left\|\hat{\mathrm{C}}_{J}^{(2 B)}(q)\right\| J_{i}\right\rangle=\sum_{a, b, j_{1}} \sum_{a^{\prime}, b^{\prime}, j_{2}}<\left[a^{\prime}, b^{\prime}\right] j_{2}\left\|\hat{\mathrm{C}}_{J}^{(2 B)}(q)\right\|[a, b] j_{1}>\Psi_{J}^{(i, f)}\left(\left[a^{\prime}, b^{\prime}\right] j_{2},[a, b] j_{1}\right)
$$

where the sums run over all initial (final) two-particle states coupled to angular momentum $j_{1}\left(j_{2}\right)$. The two-body density matrix element is written in terms of single particle creation operators, $c_{\alpha}^{\dagger}$, in the form

$$
\begin{aligned}
\left.\Psi_{J}^{(i, f)}\left(\left[a^{\prime}, b^{\prime}\right] j_{1},[a, b] j_{2}\right)\right)= & \\
& \frac{1}{[J]}<J_{i}\left\|\left[\left[c_{a^{\prime}}^{\dagger} \otimes c_{b^{\prime}}^{\dagger}\right]_{j_{2}} \otimes\left[\tilde{c}_{a} \otimes \tilde{c}_{b}\right]_{j_{1}}\right]_{J}\right\| J_{f}>
\end{aligned}
$$

with $\tilde{c}_{a}=(-1)^{j_{a}+m_{a}} c_{-a}$. (We refer the reader to Sec.IV.E in Donnelly and Sick's review article [20] for the explicit expressions of the 2B-density matrix elements in the case of one particle or one hole outside a closed shell which are those of interest for this work.)

Equation (2.17) requires the calculation of the reduced matrix element of the axial Coulomb operator between two-particle states,

$$
\left|\left[a^{\prime}, b^{\prime}\right]_{j_{1}}^{m_{1}}\right\rangle=\sum_{m_{a}, m_{b}}\left\langle j_{a} m_{a} j_{b} m_{b} \mid j_{1} m_{1}\right\rangle\left|j_{a} m_{a}\right\rangle\left|j_{b} m_{b}\right\rangle
$$

where

$$
\left\langle\vec{x} \mid j_{a} m_{a}\right\rangle=\Psi_{n k}^{\mu}(x)=\left(\begin{array}{c}
G_{n k}(x) \mathcal{Y}_{k}^{\mu}(\Omega) \\
i F_{n k}(x) \mathcal{Y}_{-k}^{\mu}(\Omega)
\end{array}\right) \exp \left\{-i E_{n_{a} l_{a}} t\right\}
$$


with

$$
\mathcal{Y}_{k}^{\mu}=\sum_{m, m_{s}}<\operatorname{lm} \frac{1}{2} m_{s} \mid j \mu>Y_{l}^{m} \chi_{\frac{1}{2}}^{m_{s}}
$$

and

$$
k_{\alpha}=(-1)^{j_{a}+l_{a}+\frac{1}{2}}\left(j_{a}+\frac{1}{2}\right)
$$

Since single particle states are obtained in co-ordinate space we transform the current accordingly.

\section{Coordinate space two-body matrix elements}

Our starting point will be the total 2B current of Eq.(2.9) (where, for convenience, all but the Lorentz indeces have been dropped.) In the Heisenberg representation we write the current in co-ordinate space in the following form,

$$
\begin{aligned}
\hat{J}_{a}^{\mu}(x)= & \frac{1}{4} g_{A} g_{\rho}^{2} \epsilon_{a b c}\left\{-g_{\nu}^{\mu}-\frac{\partial^{\mu} \partial_{\nu}}{q^{2}-m_{\pi}^{2}}\right\} \\
& \times \int d^{4} x_{1} \Delta_{\pi}^{0}\left(x-x_{1}\right) \partial_{\alpha}^{1}\left[\bar{\psi}\left(x_{1}\right) \gamma^{\alpha} \gamma^{5} \tau^{b} \psi\left(x_{1}\right)\right] \\
& \times \int d^{4} x_{2} \Delta_{\rho}^{0}\left(x-x_{2}\right)\left[-g^{\nu \eta}\right]\left[\bar{\psi}\left(x_{2}\right) \gamma_{\eta} \tau^{c} \psi\left(x_{2}\right)\right]
\end{aligned}
$$

where

$$
\Delta_{\pi, \rho}^{0}=-\int \frac{d \kappa^{0}}{2 \pi} e^{-i \kappa^{0}\left(t-t^{\prime}\right)} \frac{e^{-M_{\pi, \rho}^{\kappa^{0}}\left|\vec{x}-\vec{x}^{\prime}\right|}}{4 \pi\left|\vec{x}-\vec{x}^{\prime}\right|}
$$

and $M_{\pi, \rho}^{\kappa^{0}}=+\sqrt{m_{\pi, \rho}^{2}-\left(\kappa^{0}\right)^{2}}$. For the $\rho N N$ vertex we have worked in the t'Hooft-Feynman gauge. We have kept a soft q-dependence in the second term of the $\pi \rho \varepsilon$ vertex in (2.23) that will be dropped in actual calculations. For future use we expand the meson propagators into multipoles using,

$$
\frac{e^{-m\left|\vec{x}-\vec{x}^{\prime}\right|}}{4 \pi\left|\vec{x}-\vec{x}^{\prime}\right|}=-m \sum_{l, m_{l}}^{\infty} j_{l}\left(i m r^{\prime}{ }_{<}\right) h_{l}^{(1)}\left(i m r^{\prime}\right) Y_{l, m_{l}}^{*}(\Omega) Y_{l, m_{l}}\left(\Omega^{\prime}\right)
$$

where $j_{l}(z)$, and $h_{l}^{(1)}(z)$ are spherical Bessel functions, and $r_{<}^{\prime}\left(r_{>}^{\prime}\right)$ denotes the lesser (greater) of $|\vec{x}|$ and $\left|\vec{x}^{\prime}\right|$. 
To calculate the reduced matrix element $<\left[a^{\prime}, b^{\prime}\right] j_{2}\left\|\hat{C}_{J}^{(2 B)}(q)\right\|[a, b] j_{1}>$ we substitute the expansion (2.25) into (2.23) perform the time and angular integrations and contract the Heisenberg operator between the appropiate two-particle states. For convenience we divide the total calculation into two separate contributions stemming from each of the two terms in the $\pi \rho \varepsilon$ vertex. For the first term proportional to the time component of the metric tensor (labelled A) we arrive at an expression of the form,

$$
\begin{aligned}
& <\left[a^{\prime}, b^{\prime}\right] j_{2}\left\|\hat{C}_{J}^{A}(q)\right\|[a, b] j_{1}>=\frac{i}{4 \sqrt{\pi}} g_{A} g_{\rho}^{2} \xi_{ \pm} \sqrt{2 J+1}(-1)^{J+1} \int d r r^{2} j_{J}(q r) \\
& \times \int d r_{1} r_{1}^{2} M_{\pi}^{\omega_{a}} \sum_{l_{1}=0}^{\infty} \sqrt{2 l_{1}+1} j_{l_{1}}\left(i M_{\pi}^{\omega_{a}} r_{<}^{1}\right) h_{l_{1}}\left(i M_{\pi}^{\omega_{a}} r_{>}^{1}\right) I_{l_{1}}\left(k_{a^{\prime}},-k_{a}\right) \\
& \times\left\{\begin{array}{l}
\left(E_{a^{\prime}}-E_{a}\right)\left[G_{a^{\prime}}\left(r_{1}\right) F_{a}\left(r_{1}\right)-F_{a^{\prime}}\left(r_{1}\right) G_{a}\left(r_{1}\right)\right] \\
\left.+\left[G_{a^{\prime}}\left(r_{1}\right)\left(\frac{\stackrel{\leftrightarrow}{\partial}}{\partial r_{1}}+\frac{2}{r_{1}}\right) G_{a}\left(r_{1}\right)+F_{a^{\prime}}\left(r_{1}\right)\left(\frac{\overleftrightarrow{\partial}}{\partial r_{1}}+\frac{2}{r_{1}}\right) F_{a}\left(r_{1}\right)\right]\right\} \\
\times \int d r_{2} r_{2}^{2} M_{\rho}^{\omega_{b}} \sum_{l_{2}=0}^{\infty} \sqrt{2 l_{2}+1} j_{l_{2}}\left(i M_{\rho}^{\omega_{b}} r^{2}<\right) h_{l_{2}}\left(i M_{\rho}^{\omega_{b}} r^{2}\right)
\end{array}\right] I_{l_{2}}\left(k_{b^{\prime}}, k_{b}\right) \\
& \times\left[G_{b^{\prime}}\left(r_{2}\right) G_{b}\left(r_{2}\right)+F_{b^{\prime}}\left(r_{2}\right) F_{b}\left(r_{2}\right)\right]\left(\begin{array}{ccc}
l_{1} & l_{2} & J \\
0 & 0 & 0
\end{array}\right)\left\{\begin{array}{ccc}
j_{a^{\prime}} & j_{b^{\prime}} & j_{2} \\
j_{a} & j_{b} & j_{1} \\
l_{1} & l_{2} & J
\end{array}\right\}
\end{aligned}
$$

where $M_{\pi, \rho}^{\omega_{a, b}}=\sqrt{m_{\pi, \rho}^{2}-\left(E_{a, b}-E_{a^{\prime}, b^{\prime}}\right)^{2}}$, and

$$
I_{L}\left(k^{\prime}, k\right)=\frac{(-1)^{j^{\prime}+1 / 2}}{4 \sqrt{\pi}} \sqrt{(2 j+1)\left(2 j^{\prime}+1\right)(2 L+1)}\left[1+(-1)^{l+l^{\prime}+L}\right]\left(\begin{array}{ccc}
j^{\prime} & L & j \\
\frac{1}{2} & 0 & -\frac{1}{2}
\end{array}\right)
$$

The symbol $\xi_{ \pm}$in (2.26) denotes the matrix element of the charge-raising (or lowering) isospin current between two-particle states,

$$
\begin{aligned}
\xi_{ \pm} & =<a^{\prime} b^{\prime}\left|\frac{1}{2 i}\left[\vec{\tau}_{1} \times \vec{\tau}_{2}\right]_{ \pm}\right| a b>= \pm<a^{\prime} b^{\prime}\left|\left(\tau_{1}^{3} \tau_{2}^{ \pm}-\tau_{1}^{ \pm} \tau_{2}^{3}\right)\right| a b> \\
& = \pm\left[\left(\delta_{a^{\prime} p} \delta_{a p}-\delta_{a^{\prime} n} \delta_{a n}\right) \delta_{b^{\prime}}{ }_{n} \delta_{b_{p}^{n}}-\delta_{a^{\prime}{ }_{n}^{p}} \delta_{a_{p}^{n}}\left(\delta_{b^{\prime} p} \delta_{b p}-\delta_{b^{\prime} n} \delta_{b n}\right)\right]
\end{aligned}
$$

The second term of the axial-charge operator proportional to $q_{0} \overrightarrow{\partial_{\nu}} /\left(q^{2}-m_{\pi}^{2}\right)$ contracts with the $\gamma^{\nu}$ matrix in the $\rho N N$ vertex giving rise to a time-like and a space-like contribution. 
The first one is simply a renormalization of the matrix element of the operator $C_{J}^{A}$ just calculated and that we now redefine so that,

$$
<\left[a^{\prime}, b^{\prime}\right] j_{2}\left\|\hat{\mathrm{C}}_{J}^{A *}(q)\right\|[a, b] j_{1}>=<\left[a^{\prime}, b^{\prime}\right] j_{2}\left\|\hat{\mathrm{C}}_{J}^{A}(q)\right\|[a, b] j_{1}>\left(1-\frac{q_{0}^{2}}{q^{2}-m_{\pi}^{2}}\right)
$$

the second $\left(\mathrm{C}_{J}^{B}\right)$ results in the following expression,

$$
\begin{aligned}
& <\left[a^{\prime}, b^{\prime}\right] j_{2}|| \hat{C}_{J}^{B}(q) \|[a, b] j_{1}>=\frac{i}{4 \sqrt{\pi}} g_{A} g_{\rho}^{2} \frac{q_{0} q}{q^{2}-m_{\pi}^{2}} \xi_{ \pm}(-1)^{J} \int d r r^{2} \\
& \times \int d r_{1} r_{1}^{2} M_{\pi}^{\omega_{a}} \sum_{l_{1}=0}^{\infty} \sqrt{2 l_{1}+1} j_{l_{1}}\left(i M_{\pi}^{\omega_{a}} r_{<}^{1}\right) h_{l_{1}}\left(i M_{\pi}^{\omega_{a}} r_{>}^{1}\right) I_{l_{1}}\left(k_{a^{\prime}},-k_{a}\right) \\
& \times\left\{\left(E_{a^{\prime}}-E_{a}\right)\left[G_{a^{\prime}}\left(r_{1}\right) F_{a}\left(r_{1}\right)-F_{a^{\prime}}\left(r_{1}\right) G_{a}\left(r_{1}\right)\right]\right. \\
& \left.\quad+\left[G_{a^{\prime}}\left(r_{1}\right)\left(\frac{\stackrel{\leftrightarrow}{\partial}}{\partial r_{1}}+\frac{2}{r_{1}}\right) G_{a}\left(r_{1}\right)+F_{a^{\prime}}\left(r_{1}\right)\left(\frac{\stackrel{\partial}{\partial}}{\partial r_{1}}+\frac{2}{r_{1}}\right) F_{a}\left(r_{1}\right)\right]\right\} \\
& \times \int d r_{2} r_{2}^{2} M_{\rho}^{\omega_{b}} \sum_{l_{2}=0}^{\infty} \sqrt{2 l_{2}+1} j_{l_{2}}\left(i M_{\rho}^{\omega_{b}} r^{2}<\right) h_{l_{2}}\left(i M_{\rho}^{\omega_{b}} r_{>}^{2}\right) \\
& \times \sum_{l=0}^{\infty}(2 l+1) \frac{1}{\sqrt{\left(l+l_{2}+2\right)\left(l+l_{2}+1\right)\left(l+l_{2}\right)}} \\
& \times\left[G_{b^{\prime}}\left(r_{2}\right) F_{b}\left(r_{2}\right) H_{l, l_{2}}\left(k_{b^{\prime}},-k_{b}\right)-F_{b^{\prime}}\left(r_{2}\right) G_{b}\left(r_{2}\right) H_{l, l_{2}}\left(-k_{b^{\prime}}, k_{b}\right)\right] \\
& \times \sqrt{2} I_{l, l_{2}}^{\prime}\left(k_{b^{\prime}},-k_{b}\right) Z_{J}\left(l, l_{1}, l_{2}\right)\left\{\begin{array}{ccc}
j_{a^{\prime}} & j_{b^{\prime}} & j_{2} \\
j_{a} & j_{b} & j_{1} \\
l_{1} & l_{2} & J
\end{array}\right\}
\end{aligned}
$$

where the functions $H_{l, l_{2}}\left(k_{b^{\prime}}, k_{b}\right), I_{l, l_{2}}^{\prime}\left(k_{b^{\prime}}, k_{b}\right)$ and $Z_{J}\left(l, l_{1}, l_{2}\right)$ are:

$$
\begin{gathered}
H_{l, l_{2}}\left(k_{b^{\prime}}, k_{b}\right)=\delta_{l l_{2}} \sqrt{2}\left(k_{b^{\prime}}-k_{b}\right)+\delta_{l \neq l_{2}}\left[-k_{b}^{\prime}-k_{b}+(-1)^{\frac{1+l_{2}-l}{2}}\left(\frac{l+l_{2}+1}{2}\right)\right] \\
I_{l, l_{2}}^{\prime}\left(k_{b^{\prime}}, k_{b}\right)=\frac{(-1)^{j_{b^{\prime}}+1 / 2}}{4 \sqrt{\pi}} \sqrt{\left(2 j_{b^{\prime}}+1\right)\left(2 j_{b}+1\right)\left(2 l_{2}+1\right)}\left[1+(-1)^{l_{b^{\prime}}+l_{b}+l_{2}}\right]\left(\begin{array}{ccc}
j_{b^{\prime}} & l & j_{b} \\
\frac{1}{2} & 0 & -\frac{1}{2}
\end{array}\right)
\end{gathered}
$$




$$
\begin{aligned}
Z_{J}\left(l, l_{1}, l_{2}\right)= & \sqrt{(J+1)(2 J+3)} j_{J+1}(q r)\left(\begin{array}{ccc}
l_{1} & l_{2} & J+1 \\
0 & 0 & 0
\end{array}\right)\left\{\begin{array}{ccc}
l_{1} & l & J \\
1 & J+1 & l_{2}
\end{array}\right\} \\
& +\sqrt{J(2 J-1)} j_{J-1}(q r)\left(\begin{array}{ccc}
l_{1} & l_{2} & J-1 \\
0 & 0 & 0
\end{array}\right)\left\{\begin{array}{ccc}
l_{1} & l & J \\
1 & J-1 & l_{2}
\end{array}\right\}
\end{aligned}
$$

Finally, the sum of (2.29) and (2.30) gives the two-body matrix element of the axialcharge operator.

\section{RESULTS AND DISCUSSION}

The coupling constants used in the calculations are shown in Table [. Both sets of parameters, DH (Dirac-Hartree) and RHA (relativistic Hartree approximation), are from Ref. [21] and are not tuned specifically to any particular mass region. The scalar masses were determined by fitting the charge radius of ${ }^{40} \mathrm{Ca}$. The other masses, fixed to their experimental values are, $M=939 \mathrm{MeV}, m_{\omega}=783 \mathrm{MeV}, m_{\rho}=770 \mathrm{MeV}$ and $m_{\pi}=139$ $\mathrm{MeV}$. In the calculations we used $g_{\rho}^{2}$ from the fitting to the bulk symmetry energy $a_{4}=35$

$\mathrm{MeV}$. The intrinsic structure of the nucleons was considered via an additional $q^{2}$ dependence of the form $\left(1+q^{2} / M_{A}^{2}\right)^{-2}$ with $M_{A}=1.2 \mathrm{GeV}$.

Tables II to $\mathrm{Q}$ summarize our results. In the tables each row displays the result of the calculation of the matrix element corresponding to the multipole operator shown on the left. They are the axial Coulomb operator (Eq.2.15) and the polar-vector longitudinal

$$
\mathrm{L}_{J M}^{2 B}(q)=\int d^{3} x\left[\frac{i}{q} \vec{\nabla} M_{J}^{M}(q \vec{x})\right] \cdot \vec{J}^{2 B}(\vec{x}),
$$

and transverse electric,

$$
\mathrm{T}_{J M}^{2 B}(q)=\int d^{3} r\left[\frac{1}{q} \vec{\nabla} \times \vec{M}_{J}^{M}(q \mathbf{r})\right] \cdot \vec{J}^{2 B}(\vec{x}),
$$

operators. In the above we used the expression

$$
\vec{M}_{J}^{M}(q \vec{x})=j_{L}(q|\vec{x}|) \vec{Y}_{J L}^{M}(\hat{x}),
$$


with

$$
\vec{Y}_{J L}^{M}=\sum_{m \mu} C_{m \mu M}^{L 1 J} Y_{L}^{M}(\hat{x}) e_{\mu}
$$

the vector spherical harmonics.

Each table is divided in three columns: The first one (NR) shows the results of a onebody non-relativistic calculation and has been included, mainly, for comparative purposes. The second (DH) shows the results of: i) a one-body (IA) calculation, ii) one- plus two-body (IA+MEC) calculation and iii) the resulting increment $\left(\delta_{A}\right)$, all three obtained within the relativistic Dirac-Hatree approximation where the vacuum structure is ignored. The third column has a structure similar to the previous one but the one-loop vacuum corrections that originate due to the presence of the strong meson fields are taken into account [22].

The increment $\delta_{A}$ defined in the tables parametrizes the difference between the "effective" axial coupling constant, as determined from the analysis of the experimental transition rates, and the "free" coupling constant such that,

$$
g_{A}^{e f f}=g_{A}^{f r e e}\left(1+\delta_{A}\right) .
$$

We recall that the "best fit" value reported by Warburton in his analysis of nuclei in the $\mathrm{A}=206-212$ mass region was

$$
\delta_{A}=1.05 \pm 0.05
$$

The first thing to notice from the tables is that, as indicated above, the longitudinal and transverse electric contributions in the one-body case are substantially smaller than their Coulomb counterparts (more than a factor of ten in lead and tin; four times smaller in thallium). Based on this result we restrict the two-body calculation to the contribution of the axial charge where, on the other hand, we expect the larger contributions coming from pion exchange.

Despite not being the main thrust of this paper we devote one paragraph to comment on the one-body vector contribution where we notice the following. In the lead transition 
(table [1]), the IA result for $\hat{L}_{1}^{V}$ and $\hat{T}_{1}^{V}$ el is enhanced a barely $3 \%$ in DH (1\% in RHA) with respect to the NRIA calculation, a result that is consistent with Warburton's finding for the polar vector enhancement, namely $\delta_{V} \approx 0$. This behaviour does not maintain, however, and we find that in the thallium decay to lead the enhancement is $65 \%$ (34\% in RHA) and in tin is $23 \%$ (18\% in RHA) over the NRIA. Thus, there is a discrepancy between this calculation and the fit to the data in the heavy-mass region [1] for the vector contribution. A priori this difference could be adscribed to the simple-minded model used here to describe the structure of the states involved; however is hard to see how a refinement in this direction could reduce drastically the last two values.

Back to the analysis of the tables the next thing we notice is that, as expected, the onebody calculation is consistently larger in the Dirac-Hartree approach than in the relativistic Hartree approximation. This is due to the value of the effective mass $M^{*}$ which is smaller in RHA than in DH. In principle, there is no theoretical argument favoring the use of one to the other given that, in both cases, the model parameters are fitted independently to bulk nuclear-matter properties. For convenience, in the analysis that follows we shall limit our discussion to the RHA results.

In the Introduction we argued that in the relativistic framework pair diagrams are already taken into account in IA; hence the analysis must proceed by studying the contribution of MEC along with the IA results. Thus, the $\nu: 2 g_{9 / 2} \rightarrow \pi: 1 h_{9 / 2}$ transition in ${ }^{209} \mathrm{~Pb} \rightarrow{ }^{209} \mathrm{Bi}$ shows that some $40 \%$ of the enhancement is present at the one-body level and a further $80 \%$ shows up at the two-body level bringing their sum up to a total enhancement of $\delta_{A}=1.2$. The other transition we studied in a heavy nucleus, $\pi:\left(3 s_{1 / 2}\right)^{-1} \rightarrow \nu:\left(3 p_{1 / 2}\right)^{-1}$ in ${ }^{207} \mathrm{Tl} \rightarrow$ ${ }^{207} \mathrm{~Pb}$ gives a $30 \%$ enhancement due to one-body processes and $55 \%$ coming from the meson exchange contributions.

If we move to lighter nuclei the situation does not change much as far as the overall increment of the effective coupling constant is concerned. In the ${ }^{133} \mathrm{Sn} \rightarrow{ }^{133} \mathrm{Sb}$ decay, dominated by the single particle transition $\nu: 2 f_{7 / 2} \rightarrow \pi: 1 g_{7 / 2}$, we obtained $42 \%$ enhancement from 
IA and $33 \%$ from the MEC processes. In the oxygen region the decay ${ }^{16} \mathrm{~N}\left(0^{-}\right) \rightarrow{ }^{16} \mathrm{O}\left(0^{+}\right)$ was studied using a simple model of nuclear structure where the first excited state of ${ }^{16} \mathrm{~N}$, $J^{\pi}=0^{-}$(with an excitation energy of $120 \mathrm{keV}$ ) was described by the mixing of two particlehole configurations,

$$
\left|0^{-}\right\rangle=\sqrt{1-\lambda^{2}}\left|\left[2 s_{1 / 2} 1 p_{1 / 2}\right]_{0^{-}}\right\rangle+\lambda\left|\left[1 d_{3 / 2} 1 p_{3 / 2}\right]_{0^{-}}\right\rangle
$$

and where the mixing amplitude $\lambda$ was taken to be the typical $\lambda \approx 0.08$ [23]. In this case we obtained $48 \%$ (IA) and $42 \%$ (MEC) for the largest component, and 18\% (IA) and $10 \%$ (MEC) for the smallest.

Overall the calculated value of $\delta_{A}$ is around $20 \%$ of that determined empirically in the heavy-mass region. The ratio of one- to two-body processes, however, is not distributed uniformly in mass. As one moves towards the heavier nuclei the share between the one-body and two-body processes is shifted from the former to the latter. It is not straightforward to explain this trade off. Naively, it could be argued that the increase in the density implies a reduced effective mass and a corresponding enhancement in the nucleon propagators; one such change, however, should affect both the one-body and two-body processes in roughly the same manner. Furthermore, the argument of enhanced matrix elements due to offdiagonal operators (operators connecting upper to lower components of the spinors) it is not strictly true for $\beta$-decay transitions where the initial and final states do not coincide. The value of the matrix element depends on the overlap of initial and final wave functions and this overlap diminishes as we go up in mass in the periodic table. A case in point are the different enhancements obtained for the vector operators, $\hat{L}_{1}^{V}$ and $\hat{T}_{1}^{V}$ el, which are very different in different transitions despite being both off-diagonal. Summarizing, the results of the tables indicate a somewhat stable contribution of the one-body processes of around $40 \%$ whereas the two-body share seems to increase slightly with the mass (O:42\%; Sn:33\%; Tl:55\%; Bi:80\%).

The calculation in oxygen with a simple nuclear model gives an enhancement close to 100\%. Before the work of Haxton and Johnson [24] in 1990, this result would have been 
considered anomalous. These authors found that the usually accepted $50 \%$ enhancement of the coupling constant fell short by some $40 \%$ of the value necessary to agree with their calculation of the $\beta$-decay rate of the $120 \mathrm{keV}^{-}$state in nitrogen. This discrepancy plus the one found in heavy nuclei prompted a more careful examination of previous calculations. Presently, this discrepancy is not considered dramatic in any mass region [10] though there seems to be still some room for other effects. Our results seem to indicate a similar behaviour (so long as we stick to the RHA model and use pseudovector coupling).

Regarding the type of coupling, Table V1 illustrates the difference between pseudoscalar and pseudovector contributions for a particular transition in lead. Use of the former leads, typically, to an increment of the calculated enhancement of approximately $50 \%$ with respect to the same calculation with pseudovector coupling. This is not, per se, ground enough to preclude the use of pseudoscalar coupling. However, for the many-body problem is more efficient [11] to work with a Lagrangian with pseudovector coupling and no $\sigma \pi \pi$ term because in this form the soft-pion limits appear naturally in terms of just pions and nucleons (without the necessity of subtle cancellations with terms containing the $\sigma$ ).

One point to notice is the dependence of the MEC results on the value of $g_{\rho}^{2}$. Calculations in finite nuclei within QHD (see, for example, Refs. [16] and [22]) usually employ $g_{\rho}^{2}=65.23$ (the value used in this work) instead of $g_{\rho}^{2}=36.76$ as calculated from the decay of the $\rho$ meson. The first value is obtained fitting the symmetry energy of the empirical mass formula; this is a many-body argument which supports its use over that obtained from $\rho$ decay. However, aside from this, there are no further arguments which are strong enough to discriminate them; this ambiguity translates into a downwards $40 \%$ uncertainty in the final MEC results. It is relevant to comment that the relativistic one-body calculation is not sensitive to the value of the $\rho$ coupling constant.

A final word about core polarization. In two previous works [12,13 we investigated the isovector weak response of the core to the external perturbation in the approximation of nuclear matter and in linear response theory summing the ring diagrams to all orders in the random phase approximation (RPA) — the so-called backflow correction [25,26]. We 
found that the effects of this core polarization (of positive and negative energy nucleons) were sizable for large values of the momentum transfer (for example those typical of the muon capture processes, $q^{2} \approx 0.9 m_{\mu}^{2}$ ) but could be neglected for the small momentum transfers involved in nuclear $\beta$ decay. Thus, we do not anticipate any dramatic increase in the calculated $\delta_{A}$ beyond those presented in this work.

Summarizing, we have studied the contribution of two-body (MEC) processes to axial charge transitions for nuclei in the lead, tin and oxygen regions. We conducted calculations in Dirac-Hartree approximation (the Walecka model) and in the relativistic Hartree approximation (where the full one-nucleon-loop effects are included.) Along the paper we discussed the sensitivities of the calculation to the change in some of the couplings and of the parameters involved. With the choices described above the results we obtained indicate that oneand two-body processes enhance the matrix elements of the axial-charge operator by some $(100 \pm 20) \%$ in all three regions studied. This result agrees with the enhancement of the axial-coupling constant needed to understand theoretically a fit to eighteen first-forbidden $\beta$ decay transitions in the lead region. From the results of this calculation the presence of a heavy scalar meson to explain a physical effect gets further support.

\section{ACKNOWLEDGMENTS}

Partial financial support from the Antorchas Foundation is gratefully acknowledged. A.O.G. is fellow of the CONICET, Argentina. 


\section{REFERENCES}

[1] E. K. Warburton, Phys. Rev. Lett. 66, 1823 (1991); Phys. Rev. C44, 233 (1991).

[2] H. O. Meyer, C. Horowitz, H. Nann, P. V. Pancella, S. F. Pate, R. E. Pollock, B. Von Przewoski, T. Rinckel, M. A. Ross and F. Sperisen, Nucl. Phys. A539, 633 (1992).

[3] I.S. Towner, Comments Nucl. Part. Phys. 15, 145 (1986).

[4] M. Kirchbach, D. O. Riska and K. Tsushima, Nucl. Phys. A542, 616 (1992).

[5] I. S. Towner, Nucl. Phys. A542, 631 (1992).

[6] C. Horowitz, H. O. Meyer and D. K. Griegel, Phys. Rev. C48, 2920 (1993).

[7] J. Delorme and I.S. Towner, Nucl. Phys. A475, 720 (1987).

[8] W. Bentz, A. Arima, H. Hyuga, k. Shimizu and k. Yazaki, Nucl. Phys. A436, 593 (1985); S. Ichii, W. Bentz and A. Arima, Nucl. Phys. A464 575 (1987).

[9] P. Blunden, Nucl. Phys. A464, 525 (1987).

[10] E. K. Warburton and I. S. Towner, Phys. Reports 243, 103 (1994).

[11] B. D. Serot, Rep. Prog. Phys., 1111855 (1992).

[12] A. O. Gattone, E. D. Izquierdo and M. Chiapparini, Phys. Rev. C46, 788 (1992).

[13] E. D. Izquierdo and A. O. Gattone, Phys. Rev. C49, 2005 (1994).

[14] G. Barenboim, A. O. Gattone and E. D. Izquierdo, Phys. Rev. C48, 2537 (1993).

[15] S. Nozawa, K. Kubodera and H. Ohtsubo, Nucl. Phys. A453, 645 (1986).

[16] B. D. Serot and J. D. Walecka, in The Relativistic Nuclear Many-Body Problem, Advances in Nuclear Physics, Vol. 16. J.W. Negele and E. Vogt eds., Plenum Press, New York 1986.

[17] R.J. Furnstahl and C.E. Price, Phys. Rev C41, 1792 ( 1990). 
[18] J.D. Walecka, in Muon Physics, Vol. II, V.W. Hughes and C.S. Wu eds. Academic Press, New York 1975, p. 113.

[19] Ivanov and Truhlik, Nucl. Phys. B234 167 (1975).

[20] T. W. Donnelly and I. Sick, Rev. Mod. Phys. 56, 461 (1984).

[21] C. J. Horowitz and B. D. Serot, Phys. Lett. 140B, 181 (1984).

[22] R. J. Furnstahl and C. E. Price, Phys. Rev. C41, 1792 (1990). The same authors use this value to calculate density distributions in, Phys. Rev. C44, 895 (1991), and a slightly larger one $\left(g_{\rho}^{2}=83.30\right)$ in Phys. Rev. C40, 1398 (1989).

[23] I. S. Towner and F. C. Khanna, Nucl. Phys. A372, 331 (1981).

[24] W. C. Haxton and C. Johnson, Phys. Rev. Lett. 65, 1325 (1990).

[25] R. J. Furnstahl and B. D. Serot, Nucl. Phys. A468, 539 (1987).

[26] J. Cohen, Phys. Rev. C48, 1346 (1993). 


\section{FIGURES}

FIG. 1. a) and b): Pair graphs representing the exchange of a scalar meson. Diagrams c) and d) are those evaluated in relativistic mean-field theories (one body).

FIG. 2. A "time-ordered" diagram involving an intermediate nucleon-antinucleon pair generated by the presence of the scalar field. This diagram is included in the relativistic meson-exchange calculation.

FIG. 3. Tree-level diagrams to be considered in the calculation of two-body processes: a) $\rho \pi$, b) $\rho \pi \pi$, c) $\Delta \pi$, and d) $\Delta \pi \pi$.

FIG. 4. a) $\pi N N$, b) $\rho N N$ and c) $\rho \pi \pi$ vertices derived from the Lagrangian of QHD-II [Eq. (2.1) in the text].

FIG. 5. The weak $\pi \varepsilon$ and $\pi \rho \varepsilon$ vertices needed for the calculation and not present explicitly in the QHD-II Lagrangian. 


\section{TABLES}

TABLE I. Parameters used for the calculations presented in the text.

\begin{tabular}{|c|c|c|c|c|c|c|}
\hline & $g_{\sigma N}^{2}$ & $m_{\sigma}$ & $g_{\omega N}^{2}$ & $g_{\pi}^{2}$ & $g_{\rho}^{2}$ & $M^{*} / M$ \\
\hline MFT & 109.6 & 520 & 190.4 & 178.4 & 65.23 & 0.54 \\
\hline RHA & 54.3 & 458 & 102.8 & 178.4 & 65.23 & 0.73 \\
\hline
\end{tabular}

TABLE II. Results for the $\nu: 2 g_{9 / 2} \rightarrow \pi: 1 h_{9 / 2}$ transition in ${ }^{209} \mathrm{~Pb} \rightarrow{ }^{209} \mathrm{Bi}$. First row: results for the axial-charge operator. All the impulse approximation (IA) results, non-relativistic (NR), Dirac-Hartree (DH) and relativistic Hartree (RHA) are from reference [12]. The contribution of pion-exchange currents is summed in the columns labeled $I A+M E C$. The total increment $\delta_{A}$ is given for the two relativistic calculations. Second and third rows: same as above but for the longitudinal $\left(\hat{L}_{1}^{V}\right)$ and transverse electric $\left(\hat{T}_{1}^{V}\right.$ el $)$ operators.

\begin{tabular}{|c|c|c|c|c|c|c|c|}
\hline & $N R$ & \multicolumn{3}{|c|}{$D H$} & \multicolumn{3}{|c|}{$R H A$} \\
\hline & $I A$ & $I A$ & $I A+M E C$ & $\delta_{A}$ & $I A$ & $I A+M E C$ & $\delta_{A}$ \\
\hline$\hat{C}_{0}^{A}$ & $1.12 \times 10^{-1}$ & $1.98 \times 10^{-1}$ & $3.12 \times 10^{-1}$ & 1.77 & $1.58 \times 10^{-1}$ & $2.48 \times 10^{-1}$ & 1.2 \\
\hline$\hat{L}_{1}^{V}$ & $5.61 \times 10^{-3}$ & $5.77 \times 10^{-3}$ & & & $5.69 \times 10^{-3}$ & & \\
\hline$\hat{T}_{1}^{V e l}$ & $7.93 \times 10^{-3}$ & $8.16 \times 10^{-3}$ & & & $8.05 \times 10^{-3}$ & & \\
\hline
\end{tabular}

TABLE III. Same as Table П for the transition ${ }^{207} \mathrm{Tl}\left(1 / 2^{+}\right) \rightarrow{ }^{209} \mathrm{~Pb}\left(1 / 2^{-}\right)$

\begin{tabular}{c|c|ccr|ccc}
\hline \hline & $N R$ & \multicolumn{2}{|c|}{$D H$} & \multicolumn{2}{|c}{$R H A$} & \\
\hline & $I A$ & $I A$ & $I A+M E C$ & $\delta_{A}$ & $I A$ & $I A+M E C$ & $\delta_{A}$ \\
$\hat{C}_{0}^{A}$ & $-0.75 \times 10^{-1}$ & $-1.15 \times 10^{-1}$ & $-1.60 \times 10^{-1}$ & 1.12 & $-0.97 \times 10^{-1}$ & $-1.37 \times 10^{-1}$ & 0.81 \\
$\hat{L}_{1}^{V}$ & $2.01 \times 10^{-2}$ & $3.33 \times 10^{-2}$ & & & \\
$\hat{T}_{1}^{V e l}$ & $2.85 \times 10^{-2}$ & $4.71 \times 10^{-2}$ & & & $2.71 \times 10^{-2}$ & \\
\hline \hline
\end{tabular}


TABLE IV. Same as Table $\llbracket$ for the transition ${ }^{133} \mathrm{Sn}\left(7 / 2^{-}\right) \rightarrow{ }^{133} \mathrm{Sb}\left(7 / 2^{+}\right)$

\begin{tabular}{c|c|ccr|ccc}
\hline \hline & $N R$ & \multicolumn{2}{|c|}{$D H$} & \multicolumn{2}{|c}{$R H A$} \\
\hline & $I A$ & $I A$ & $I A+M E C$ & $\delta_{A}$ & $I A$ & $I A+M E C$ & $\delta_{A}$ \\
$\hat{C}_{0}^{A}$ & $0.98 \times 10^{-1}$ & $1.69 \times 10^{-1}$ & $2.22 \times 10^{-1}$ & 1.26 & $1.42 \times 10^{-1}$ & $1.70 \times 10^{-1}$ & 0.73 \\
$\hat{L}_{1}^{V}$ & $0.66 \times 10^{-2}$ & $0.81 \times 10^{-2}$ & & & $0.78 \times 10^{-2}$ & \\
$\hat{T}_{1}^{V e l}$ & $0.93 \times 10^{-2}$ & $1.15 \times 10^{-2}$ & & & $1.10 \times 10^{-2}$ & \\
\hline \hline
\end{tabular}

TABLE V. Same as Table II for the transition ${ }^{16} \mathrm{~N}\left(0^{-}\right) \rightarrow{ }^{16} \mathrm{O}\left(0^{+}\right)$except that the one-body longitudinal $\left(\hat{L}_{1}^{V}\right)$ and transverse electric $\left(\hat{T}_{1}^{V \text { el }}\right)$ contributions have been substituted by the $J=3 / 2$ contribution from the transition due to the second term in Eq. (3.6)

\begin{tabular}{c|c|ccc|ccc}
\hline \hline & $N R$ & \multicolumn{2}{|c|}{$D H$} & \multicolumn{3}{|c}{$R H A$} \\
\hline & $I A$ & $I A$ & $I A+M E C$ & $\delta_{A}$ & $I A$ & $I A+M E C$ & $\delta_{A}$ \\
$\hat{C}_{0}^{A} j=1 / 2$ & $3.95 \times 10^{-2}$ & $5.97 \times 10^{-2}$ & $7.90 \times 10^{-2}$ & 1.35 & $5.86 \times 10^{-2}$ & $7.51 \times 10^{-2}$ & 0.90 \\
$\hat{C}_{0}^{A} j=3 / 2$ & $-1.31 \times 10^{-1}$ & $-1.64 \times 10^{-1}$ & $-1.78 \times 10^{-1}$ & 0.36 & $-1.56 \times 10^{-1}$ & $-1.68 \times 10^{-1}$ & 0.28 \\
\hline \hline
\end{tabular}

TABLE VI. Comparison of the MEC results using pseudovector (PV) coupling and pseudoscalar plus the $\sigma \pi \pi$ term for the axial charge operator. The results correspond to the $\nu: 2 g_{9 / 2} \rightarrow \pi: 1 h_{9 / 2}$ transition in ${ }^{209} \mathrm{~Pb} \rightarrow{ }^{209} \mathrm{Bi}$ and the labels are the same as in Table $\mathbb{1}$. The second row shows the enhancement with respect to the $N R$ calculation.

\begin{tabular}{c|cccccc}
\hline \hline$N R$ & \multicolumn{3}{c|}{$D H$} & \multicolumn{3}{c}{$R H A$} \\
\hline$I A$ & $I A$ & $M E C(P V)$ & $M E C(P S)$ & $I A$ & $M E C(P V)$ & $M E C(P S)$ \\
\hline$-1.23 \times 10^{-1}$ & $-1.98 \times 10^{-1}$ & $-1.14 \times 10^{-1}$ & $-2.63 \times 10^{-1}$ & $-1.57 \times 10^{-1}$ & $-8.97 \times 10^{-2}$ & $-2.14 \times 10^{-1}$ \\
& 0.60 & 0.90 & 2.14 & 0.28 & 0.72 & 1.72 \\
\hline \hline
\end{tabular}


This figure "fig1-1.png" is available in "png" format from: http://arxiv.org/ps/nucl-th/9504009v1 
This figure "fig1-2.png" is available in "png" format from: http://arxiv.org/ps/nucl-th/9504009v1 
This figure "fig1-3.png" is available in "png" format from: http://arxiv.org/ps/nucl-th/9504009v1 
This figure "fig1-4.png" is available in "png" format from: http://arxiv.org/ps/nucl-th/9504009v1 
This figure "fig1-5.png" is available in "png" format from: http://arxiv.org/ps/nucl-th/9504009v1 